\title{
Circulating exosomal microRNAs as novel potential detection biomarkers in pancreatic cancer
}

\author{
LUN WU ${ }^{1}$, WEN-BO ZHOU ${ }^{2}$, JIAO ZHOU ${ }^{3}$, YING WEI $^{4}$, HONG-MEI WANG $^{5}$, XIAN-DE LIU $^{6}$, \\ XIAO-CHUN CHEN ${ }^{6}$, WEI WANG ${ }^{1}$, LIN YE ${ }^{1}$, LI CHAO YAO ${ }^{1}$, QIN-HUA CHEN ${ }^{7}$ and ZHI-GANG TANG ${ }^{1}$ \\ ${ }^{1}$ Department of Pancreatic Surgery, Renmin Hospital of Wuhan University, Wuhan, Hubei 430060; \\ Departments of ${ }^{2}$ Hepatobiliary Surgery and ${ }^{3}$ Urology; ${ }^{4}$ Clinical Laboratory; ${ }^{5}$ Liver Surgery Institute of \\ The Experiment Center of Medicine, Dongfeng Hospital, Hubei University of Medicine; \\ ${ }^{6}$ Department of General Surgery, People's Hospital of Zhu Shan; ${ }^{7}$ Hubei Key Laboratory of \\ Wudang Local Chinese Medicine Research, Experiment Center of Medicine, \\ Hubei University of Medicine, Shiyan, Hubei 442001, P.R. China
}

Received November 13, 2019; Accepted April 28, 2020

DOI: $10.3892 / \mathrm{ol} .2020 .11691$

\begin{abstract}
Circulating exosomal microRNAs (ex-miRNAs) are reflective of the characteristics of the tumor and are valuable biomarkers in different types of tumor. In addition, miRNAs serve important roles in tumor progression and metastasis. The present study aimed to investigate the circulating ex-miRNA-21 and miRNA-210 as novel biomarkers for patients with pancreatic cancer (PC). For this purpose, serum ex-miRNAs were extracted from the serum of patients with PC $(n=30)$ and chronic pancreatitis $(C P)(n=10)$ using an RNA isolation kit. For exosome identification in serum, transmission electron micrographs were used to determine crystalline structure, western blotting was used to identify exosomal markers, and NanoSight was used for nanoparticle characterization. The relative expression levels of ex-miRNAs were quantified using quantitative PCR and compared between patients with PC and CP. The expression levels of both ex-miRNA-21 and miRNA-210 were significantly higher in patients with PC compared with patients with $\mathrm{CP}$ (both $\mathrm{P}<0.001$ ). However, no significant difference in the relative serum levels of free miR-21 and miR-210 was observed between the 2 groups of patients (both $\mathrm{P}>0.05$ ). ex-miRNA-21 and miRNA-210 were associated with tumor stage, as well as other factors. The diagnostic potential of ex-miRNA-21 and miRNA-210 levels was 83 and $85 \%$, respectively. In addition, when ex-miRNA and serum carbohydrate antigen 19-9 expression levels were combined, the accuracy increased to $90 \%$. The present study
\end{abstract}

Correspondence to: Professor Zhi-Gang Tang, Department of Pancreatic Surgery, Renmin Hospital of Wuhan University, 99 Hubei Zhang Road, Wuhan, Hubei 430060, P.R. China

E-mail: tzg7031@163.com

Key words: exosome, microRNA, circulating biomarker, pancreatic cancer identified that serum ex-miRNAs, miRNA-21 and miRNA-210 may be of value as potential biomarkers and therapeutic targets for the diagnosis and treatment of PC.

\section{Introduction}

Pancreatic cancer (PC) is one of the most highly malignant cancers, with a mean 5-year survival rate of $<9 \%$ in the United States $(1,2)$. According to a previous report, PC is responsible for 227,000 deaths annually worldwide due to its high recurrence rate, asymptomatic onset and $<5 \%$ resectable localized tumors (3). Unfortunately, early diagnostic and effective treatment strategies are lacking for PC and there is an urgent need to explore diagnostic tools with high sensitivity, specificity and repeatability (4). Thus, there is an urgent need to investigate the underlying molecular mechanisms of PC and identify novel diagnostic biomarkers for the disease. Identifying a minimally invasive/non-invasive and sensitive diagnostic method has recently become a popular topic of research and there has been some progression in the study of serum markers $(5,6)$. Further elucidation of the associated biological processes involved is crucial as it may uncover novel potential biomarkers for early diagnosis of PC.

MicroRNAs (miRNAs) are 18-22 nucleotides in length, endogenous non-coding single-stranded RNAs that regulate gene expression by binding to the 5 ' or 3'-untranslated regions post-transcriptionally or to the open reading frames $(7,8)$. mRNA or protein is degraded or translation suppressed by miRNAs through this interference process (9). Numerous studies have demonstrated that miRNAs are involved in a variety of malignancies, such as PC, lung, breast and prostate cancer (10-12). In particular, the clinical applications of miRNAs have been investigated as biomarkers for early diagnosis and treatment of tumors, clinical response and histological classification (13). Due to the repeatability and ease of sample collection, circulating miRNAs are currently being investigated as non-invasive biomarkers in the early diagnosis of different types of cancer (14). Valadi et al (15) first extracted 
and verified miRNAs in exosomes, and demonstrated that exosomes are secreted by different types of cells and may be used for transporting proteins, mRNAs and miRNAs to target cells. The study of miRNAs in exosomes is becoming a new research focus. Furthermore, it was revealed that exosomal (ex)-miRNAs may play crucial roles in biological processes such as tumor progression, cell proliferation, invasion, metastasis, apoptosis and differentiation (16-19), and may be of value as novel serum biomarkers for the early diagnosis of cancer, such as PC.

Exosomes originate from internal multivesicular bodies and are membranous vesicles with a diameter of 30-120 nm (20,21). Exosomes have been identified in serum, plasma, breast milk and other human bodily fluids (22). Recent findings have reported that ex-miRNAs contain miRNA, mRNA and cell-specific proteins, and have been used as diagnostic biomarkers in tumor cells as they may reflect genetic information and molecular signatures of the various cells of origin (23-25). Tumor cell derived exosomes contain tumor-specific miRNAs and their roles are emerging in malignant progression (26). However, to the best of our knowledge, the role of circulating ex-miRNAs in PC has not yet been extensively investigated.

Previous studies have demonstrated that miRNA-21 and miRNA-210 are associated with a poor prognosis in PC; however, the findings of these studies were based on tumor tissues and a small sample size limited their clinical significance $(27,28)$. Furthermore, there are currently few reports on the significance of circulating ex-miRNAs as diagnostic markers of PC (29-31). The aim of the present study was to investigate the diagnostic relevance of two circulating serum ex-miRNAs, miRNA-21 and miRNA-210 as novel serological biomarkers for PC.

\section{Materials and methods}

Patients. A total of 40 patients at the Renmin Hospital of Wuhan University (Wuhan, China) were enrolled between March 2018 and August 2019 in the present study and their clinicopathological characteristics were evaluated. Among the patients, 30 had PC and 10 had CP. A diagnosis of PC was made by pathology or cytology, while a diagnosis of chronic pancreatitis $(\mathrm{CP})$ was made by pathology or clinical criteria based on the World Health Organization Classification of Tumours of the Digestive System (32). The enrollment criteria included subjects who had not received neoadjuvant chemoradiotherapy. Patients were excluded if the diagnosis of PC was not pathologically confirmed. Cases were staged according to the modified European Neuroendocrine Tumor Society Tumor-Node-Metastasis (TNM) staging system (33). The serum ex-microRNAs in patients with PC were detected prior to surgical resection. The study protocol was approved by the Ethics Committee of Renmin Hospital of Wuhan University (approval no. 20180212; Wuhan, China). Written informed consent was provided by all patients.

Isolation and identification of exosomes from the serum of patients with $P C$. Blood samples $(5-6 \mathrm{ml})$ were collected from all patients and centrifuged at $500 \mathrm{x} \mathrm{g}$ for $5 \mathrm{~min}$ at $4^{\circ} \mathrm{C}$. The supernatants were preserved at $-80^{\circ} \mathrm{C}$ until further use. Exosomes were isolated from serum samples using the Exosome extraction kit (System Biosciences). Briefly, $500 \mu 1$ of serum was mixed with $120 \mu$ l ExoQuick solution and incubated at $4^{\circ} \mathrm{C}$ for $30 \mathrm{~min}$. The mixed solution (ExoQuick/serum) was centrifuged at $12,000 \mathrm{x} \mathrm{g}$ for $2 \mathrm{~min}$. for obtaining exosome pellets. Transmission electron microscopy (TEM; cat. no. HT7800; Hitachi High-Technologies Corporation; HC mode, x200-200,000; HR mode, x4,000-600,000; LowMag mode, $x 50-1,000)$ was used to visualize and verify the exosomes that were extracted from the serum. The nanoparticle size distribution and concentration of vesicles were analyzed on the NanoSight LM10-HS instrument (NanoSight, Ltd.) using NTA 3.0 software (34).

Western blotting. The exosomes marker proteins were detected by western blotting. Total protein of exosomes was extracted with a cell lysis buffer (cat. no. 9803; Cell Signaling Technology, Inc.) supplemented with $1 \mathrm{mM}$ protease inhibitor cocktail (cat. no. 5871; Cell Signaling Technology, Inc.). Equal amounts of extracted protein $(0.5 \mu \mathrm{g} / \mathrm{lane})$ were separated by $10 \%$ SDS-PAGE and transferred to polyvinylidene difluoride (PVDF) membranes (Thermo Fisher Scientific, Inc.). The blots were blocked with $5 \%$ non-fat milk at $37^{\circ} \mathrm{C}$ for $2 \mathrm{~h}$ prior to incubation overnight at $4^{\circ} \mathrm{C}$ with primary antibodies anti-cluster of differentiation (CD)63 (1:500; cat. no. ARG57952; Arigo Biolaboratories, Corp.), antitumor susceptibility gene (TSG)101 (1:1,000; cat. no. ab125011; Abcam) and anti-CD81 (1:500; cat. no. MA1-10290; Thermo Fisher Scientific, Inc.). GAPDH (1:5,000; cat. no. AF7021; Affinity Biosciences) was used as the internal loading control. The blots were incubated with horseradish peroxidase-conjugated anti-IgG secondary antibodies (1:5,000; cat. no. ANT022; Wuhan AntGene Biotechnology Co., Ltd.) at room temperature for $2 \mathrm{~h}$, and subsequently developed using an enhanced chemiluminescence kit (cat. no. 34095; Thermo Fisher Scientific, Inc.) and semi-quantified using the Quantity One software version 4.62 (Bio-Rad Laboratories Inc.).

miRNA extraction. miRNA extraction from the isolated exosomes was performed using a miRNeasy Mini kit (Qiagen $\mathrm{GmbH}$ ) according to the manufacturer's instructions. The final volumes of RNA were standardized by dilution with $30 \mu 1$ nuclease-free water (cat. no. ST876; Beyotime Institute of Biotechnology). The RNA concentration in $2 \mu \mathrm{l}$ was quantified using a Microvolume UV-Vis Spectrophotometer (cat. no. ND-ONE-W; Thermo Fisher Scientific, Inc.).

Measurement of miRNA levels using reverse transcriptionquantitative (RT-q) PCR. In order to investigate ex-miRNAs, miRNA-21 and miRNA-210 were selected $(34,35)$. Total RNA extraction from the isolated exosomes was performed as aforementioned. The RNA was reverse-transcribed using the TaqMan MicroRNA Reverse Transcription kit (Qiagen $\mathrm{GmbH}$ ) at $37^{\circ} \mathrm{C}$ for $60 \mathrm{~min}$ and $94^{\circ} \mathrm{C}$ for $5 \mathrm{~min}$. qPCR was performed using the TaqMan miRNA kit according to the manufacturer's protocol (Qiagen $\mathrm{GmbH}$ ) and $\mathrm{Cq}$ values were calculated. The primers used were as follows: miR-21 forward, 5'-CGCTAGCTTATCAGACTG-3' and reverse, 5'-GAGCAG GCTGGAGAA-3'; miR-210 forward, 5'-ACACTCCAGCTG GGCTGTGCGTGTG-3' and reverse, 5'-CAACTGGTGT 
Table I. Comparison of mean values of age, sex, location, stage, T factor and CA19-9 levels between patients with PC and CP.

\begin{tabular}{lccc}
\hline Characteristics & PC & CP & P-value \\
\hline Mean age (range), years & $62.0(30-80)$ & $50.5(34-80)$ & 0.17 \\
Sex, male/female, $\mathrm{n}$ & $18 / 12$ & $8 / 2$ & 0.64 \\
Location, head/body/tail, n & $12 / 8 / 10$ & - & NA \\
Stage, /IA/IB/IIA/IIB/III/IV & $1 / 0 / 1 / 4 / 14 / 2 / 8$ & - & NA \\
T factor, cis/1/2/3/4 & $1 / 1 / 4 / 20 / 4$ & - & NA \\
Mean CA19-9 (range), U/ml & $104.3(0.5-15,00.0)$ & $15.0(4.0-100.5)$ & $0.01^{\text {b }}$
\end{tabular}

${ }^{a}$ TNM staging system (33). ${ }^{b} \mathrm{P}<0.05$. Student's t-test and $\chi^{2}$ test were used for statistical analysis. PC, pancreatic carcinoma; $\mathrm{CP}$, chronic pancreatitis; CA19-9, carbohydrate antigen 19-9; T, tumor; NA, not applicable.

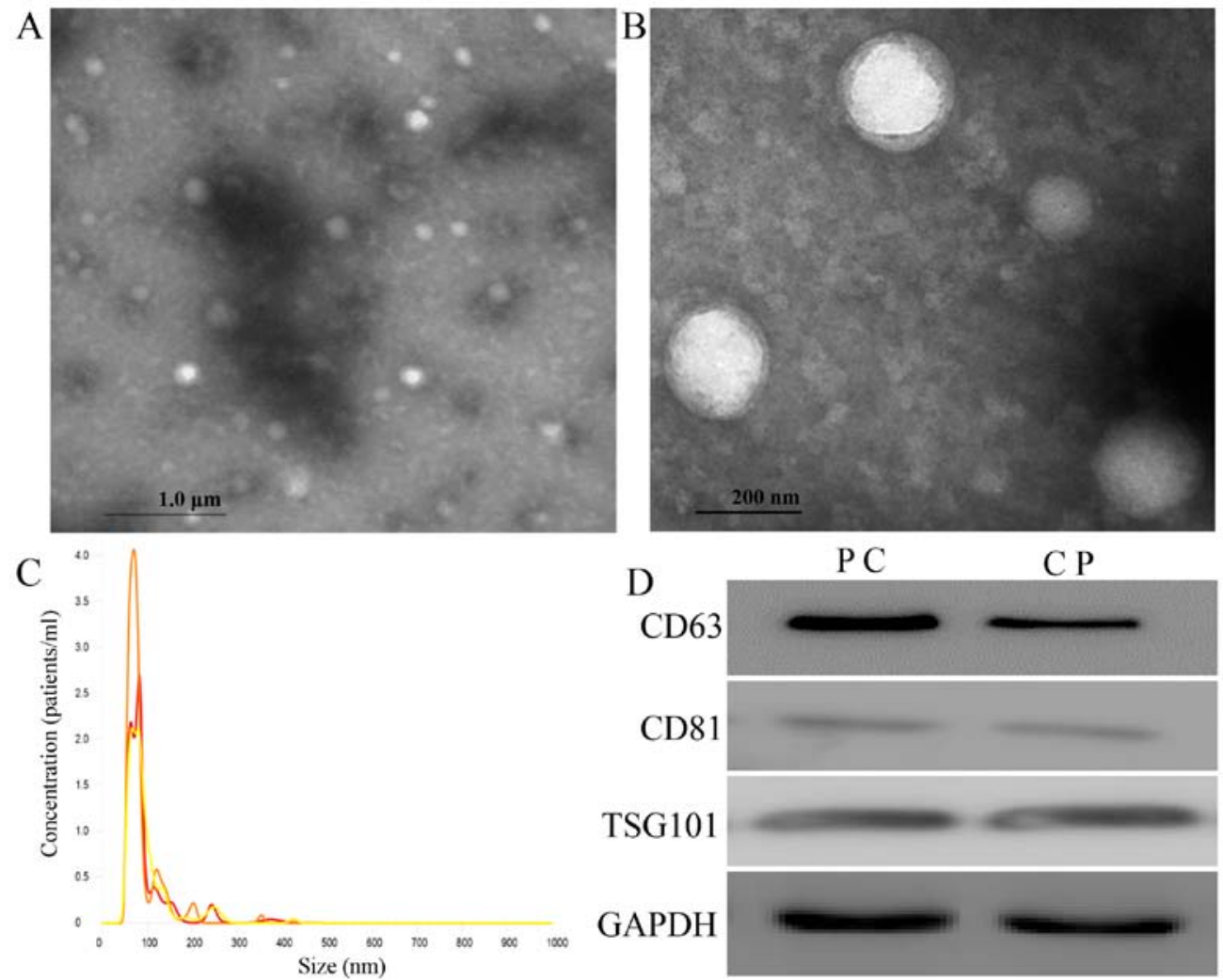

Figure 1. Transmission electron microscopy, NanoSight and western blot analysis were used to examine circulating serum exosomes. (A and B) Exosomes are represented as small vesicles in the serum imaged using transmission electron microscopy with the negative stain method. (C) Exosomes isolated from serum were analyzed using a NanoSight. The particle size distribution and concentration were $93 \mathrm{~nm}$ and $1.3 \times 10^{9}$ particles/ml, respectively. (D) Exosomes extracted from serum were analyzed by western blotting using anti-CD63, -CD81 and -TSG101 antibodies. CD63, CD81 and TSG101 were present in the exosomes. PC, pancreatic cancer; $\mathrm{CP}$, chronic pancreatitis; $\mathrm{CD}$, cluster of differentiation; TSG, tumor susceptibility gene.

CGTGGAGTCG-3'. PCR amplifications were performed in triplicate for each sample. The thermocycling conditions used were as follows: $95^{\circ} \mathrm{C}$ for $15 \mathrm{~min}$, followed by 45 cycles at $94^{\circ} \mathrm{C}$ for $15 \mathrm{sec}$ and finally $55^{\circ} \mathrm{C}$ for $30 \mathrm{sec}$. The relative miRNA expression values were normalized to cel-miR-39 $(36,37)$ and calculated using the $2^{-\Delta \Delta \mathrm{Cq}}$ method (38).

Statistical analysis. Data were statistically analyzed using SPSS version 20.0 (IBM Corp.). Data are presented as the median (interquartile range) or $\mathrm{n}(\%)$ values as appropriate. The association between miRNA expression levels and clinicopathological features was examined by $\chi^{2}$ or Fisher's exact test. Comparisons of continuous data were made using the unpaired Student's t-test. The optimal cut-off was established as the mean threshold or critical value that maximized the sum of sensitivity and specificity using time-dependent receiver operating characteristic (ROC) curves and the areas under curves (AUC). The diagnostic value of the candidate miRNAs was evaluated by calculating specificity and sensitivity. The cut-off points were determined using the Youden index and were 0.09 and 0.0012 for miRNA-21 and miRNA-210, respectively. AUCs for miRNAs and CA19-9 were compared using Z tests. $\mathrm{P}<0.05$ was considered to indicate a statistically significant difference. 


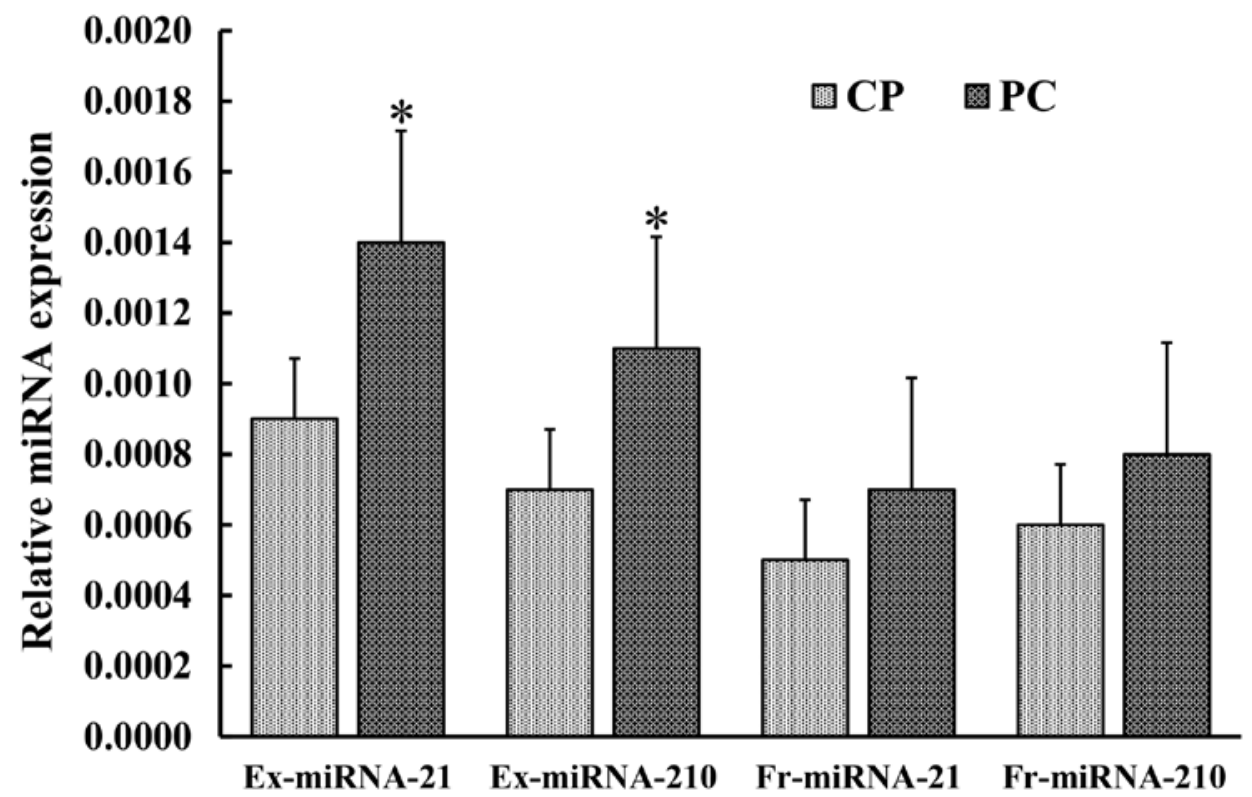

Figure 2. Expression level of miRNA-21 and miRNA-210 in the serum of patients with PC and CP. Compared with patients with CP, the expression levels of Ex-miRNA-21 and Ex-miRNA-210 were significantly higher in patients with PC. "P<0.01. CP, chronic pancreatitis; PC, pancreatic carcinoma; Ex-miRNA, exosomal microRNA; Fr-miRNA, free microRNA; cel, cellular.

\section{Results}

Patient characteristics. Clinicopathological characteristics of the patients are presented in Table I. The mean age was 62 years for patients with PC and 50.5 years for patients with CP. The median diameter of the tumor was $4.2 \mathrm{~cm}$ in patients with PC (data not shown). The mean level of serum carbohydrate antigen (CA) 19-9 was significantly higher in patients with PC compared with patients with $\mathrm{CP}(\mathrm{P}<0.05)$, whereas no statistically significant differences were observed in sex or age (Table I).

Identification of circulating serum exosomes. Exosomes were collected and observed using TEM (Fig. 1A and B) in accordance with the characteristics of exosomes previously described (39). Numerous small vesicles were observed by a NanoSight in the range of 50-100 $\mathrm{nm}$ and the size and overall concentration were $\sim 93 \mathrm{~nm}$ and $1.3 \times 10^{9}$ particles $/ \mathrm{ml}$, respectively (Fig. 1C). Known exosome-specific markers, such as CD63, CD81 and TSG101 were identified in the vesicles by western blotting, and the exosome vesicles obtained from patients with PC were compared with those from patients with $\mathrm{CP}$; it was observed by western blot analysis that the expression of these markers in patients with PC was stronger than that in patients with $\mathrm{CP}$ (Fig. 1D).

Expression level of serum ex- and serum fr-circulating miRNAs in patients with $P C$ and $C P$ by RT-qPCR. The expression levels of serum ex-miRNA-21 and miRNA-210 were significantly higher in patients with $\mathrm{PC}(\mathrm{P}<0.01$; Fig. 2$)$. There was a significant difference between the AUC values for ex-miRNA-21 and fr-miRNA-21 (P=0.003; Fig. 3A). Similarly, there was a significant difference between the AUC values for ex-miRNA-210 and fr-miRNA-210 ( $\mathrm{P}=0.009$; Fig. 3B).

Comparison of the diagnostic values of ex-miRNAs, serum circulating miRNAs and CA19-9 quantitation. Diagnostic values of serum circulating free or exosomal miRNA-21 and miRNA-210 were investigated. The AUC values for ex-miRNA-21 and ex-miRNA-210 levels were significantly higher compared with those for serum CA19-9 levels (both $\mathrm{P}=0.038$; Fig. $3 \mathrm{C}$ and D). The cut-off values for ex-miRNA-21 and ex-miRNA-210 levels were determined and used to stratify patients into two groups (positive or negative) for each ex-miRNA. The accuracy of ex-miRNA-21 (83\%) and ex-miRNA-210 (85\%) were superior compared with serum fr-miRNA-21 (73\%) and fr-miRNA-210 (78\%) (Table II). When combining the results of ex-miRNA-21 and ex-miR-210, the accuracy, specificity and sensitivity were 90,80 and $93 \%$, respectively (Table II). Taking the positive results of either ex-miRNA-21/ex-miRNA-210 levels or serum cytology as the criterion for diagnosis of $\mathrm{PC}$, the specificity of the combined test (with CA19-9) and sensitivity was 90\% (Table II).

Serum ex-miRNA-21 and miRNA-210 levels are associated with multiple prognostic factors of PC. Associations between the relative levels of these miRNA-21 and miRNA-210 and clinical characteristics were evaluated. High and low expression levels of miRNA-21 and miRNA-210 were based on the cut-off values of 0.09 and 0.0012 , respectively. The expression levels of miRNA-21 and miRNA-210 were associated with the Tumor-Node (TN) stage (31), and the levels of these miRNAs were closely associated with advanced disease $(\mathrm{P}<0.05)$ and other prognostic factors, including TN stage, age, treatment history, location, CA19-9, size of tumor and CRP, while they were not associated with sex and M stage (Table III).

\section{Discussion}

Recently, the abnormal expression of serum ex-miRNAs has emerged as a potential novel biomarker of tumor diagnosis and progression due to their stability in plasma/serum and their specific expression profiles which reflect the gene information and biolog- 
A

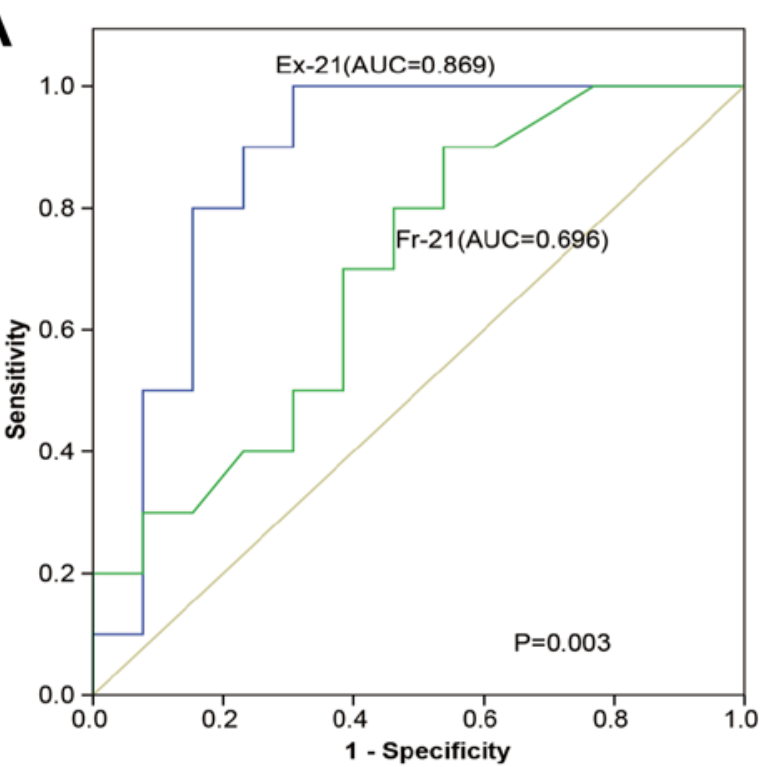

C

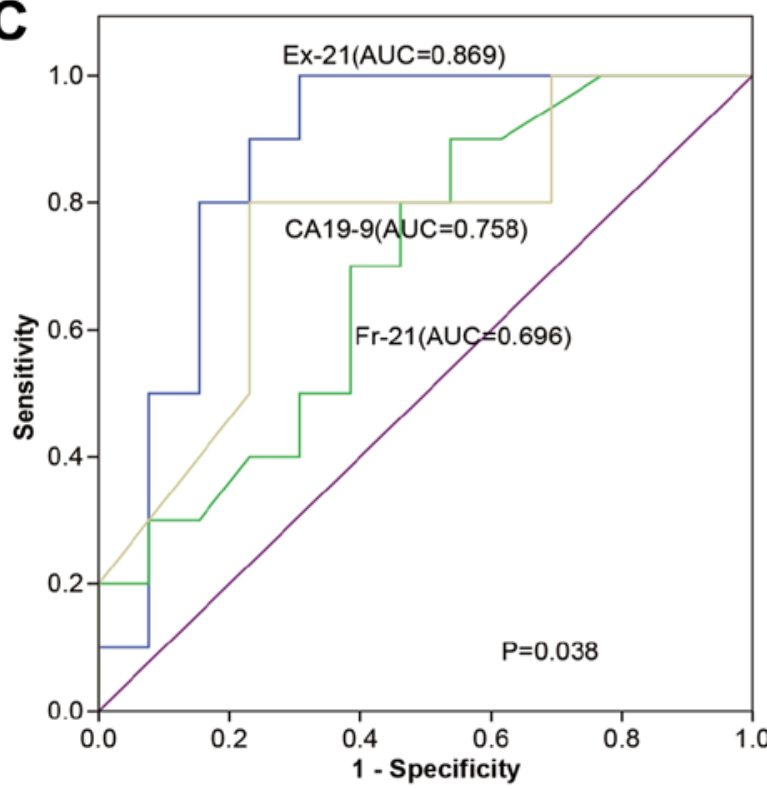

B

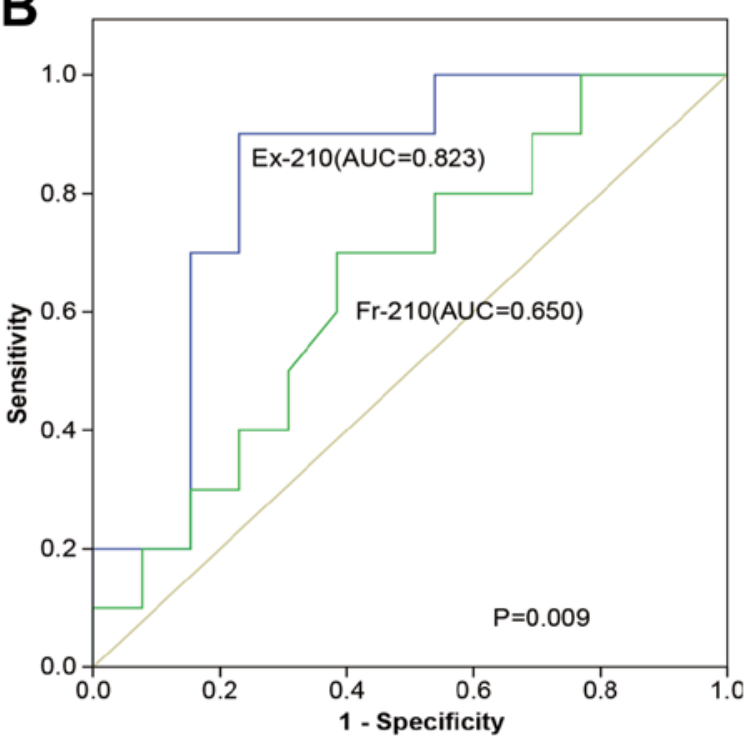

D

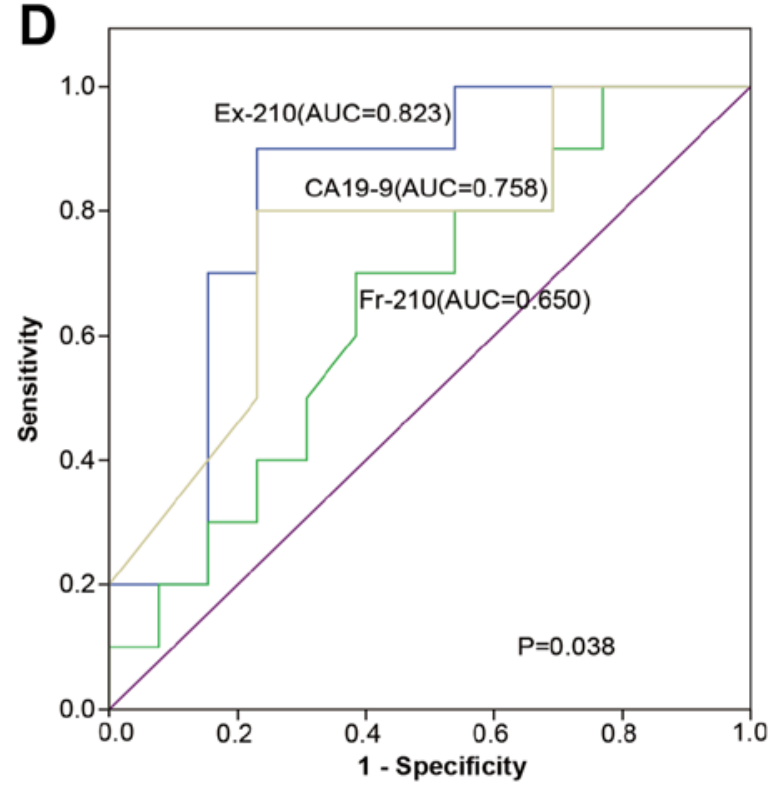

Figure 3. Receiver operating characteristic curve analysis was performed to distinguish the diagnostic value of miRNA and serum CA19-9. (A) Diagnostic value of Ex-21 levels compared with levels of Fr-21, with AUC values of 0.869 and 0.696, respectively. (B) Diagnostic value of Ex-210 level compared with levels of Fr-210, with AUC values of 0.823 and 0.650, respectively. (C) Diagnostic value of Ex-21 level and CA19-9 level, with AUC values of 0.869 and 0.758, respectively. (D) Diagnostic value of Ex-210 level and CA19-9 level, with AUC values of 0.823 and 0.758, respectively. AUC, area under the curve; Ex-21, exosomal miRNA-21; Ex-210, exosomal miRNA-210; Fr-21, free-miRNA-21; Fr-210 free-miRNA-210; CA19-9, carbohydrate antigen 19-9.

ical properties of tumor cells $(40,41)$. These serum ex-miRNAs may be used as non-invasive, specific and sensitive biomarkers for the diagnosis and prognosis of tumors (42). Alterations of circulating miRNAs have been investigated in patients with PC (43). However, studies on serum ex-miRNAs in PC are lacking. In the present study, the levels of fr- and ex-miRNA-21 and miRNA-210 were investigated in patients with PC.

Numerous studies have indicated that serum ex-miRNAs are associated with tumor-derived microRNAs and that these miRNAs may be of value as diagnostic biomarkers for cancer $(44,45)$. In the present study, compared with patients with $\mathrm{CP}$, the expression levels of serum ex-miRNA-21 and miRNA-210 were significantly higher in patients with PC. In contrast, the expression levels of serum fr-miRNA-21 and miRNA-210 did not differ significantly between patients with
$\mathrm{CP}$ and PC. Whole circulating peripheral blood contains other miRNAs in addition to ex-miRNAs, which are easily degraded probably because these miRNAs are not enclosed in exosomes $(46,47)$. In the present study, it was hypothesized that ex-miRNAs rather than serum fr- miRNAs were preferable and may serve as new biomarkers for PC. In fact, serum ex-miRNAs originating from tumor-derived miRNAs may be used as novel diagnostic biomarkers (48). In addition, ex-miRNAs in the serum or plasma and other bodily fluids may be more stable and valuable as biomarkers for PC detection compared with serum fr-miRNAs.

miRNA-21 is upregulated in different types of tumors, such as esophageal squamous cell carcinoma and breast, lung and liver cancer, and targets tumor-suppressive mRNAs $(49,50)$. It was demonstrated that overexpression of serum ex-miRNA-21 
Table II. Diagnostic value of Ex-miR or serum Fr -miRNA21 and -miRNA210 level for patients with PC calculated via receiver operating characteristic curve analysis.

\begin{tabular}{lccccccccc}
\hline Ex-miR & $\mathrm{TP}, \mathrm{n}$ & $\mathrm{FN}, \mathrm{n}$ & $\mathrm{FP}, \mathrm{n}$ & $\mathrm{TN}, \mathrm{n}$ & Sensitivity, \% & Specificity, \% & Accuracy, \% & PPV, \% & NPV, \% \\
\hline Ex-miR-21 & 24 & 6 & 1 & 9 & 80 & 90 & 83 & 96 & 60 \\
Ex-miR-210 & 25 & 5 & 1 & 9 & 83 & 90 & 85 & 96 & 64 \\
Ex-miR-21/210 & 28 & 2 & 2 & 8 & 93 & 80 & 90 & 93 & 80 \\
Fr-miR21 & 22 & 8 & 3 & 7 & 73 & 70 & 73 & 88 & 47 \\
Fr-miR210 & 23 & 7 & 2 & 8 & 76 & 80 & 78 & 92 & 53 \\
Ex-miR-21/CA19-9 & 27 & 3 & 1 & 9 & 90 & 90 & 90 & 96 & 75 \\
Ex-miR-210/CA19-9 & 27 & 3 & 1 & 9 & 90 & 90 & 90 & 96 & 75 \\
\hline
\end{tabular}

TP, true positive; FN, false negative; FP, false positive; TN, true negative; PPV, positive predictive value; NPV, negative predictive value; Ex-miR, exosomal microRNA, Fr-miR, free microRNA; PC, pancreatic carcinoma.

Table III. Associations between expression levels of both serum ex-miRNA-21 and ex-miRNA-210 level and clinical characteristics in patients with pancreatic cancer $(\mathrm{n}=30)$.

\begin{tabular}{|c|c|c|c|c|c|c|}
\hline \multirow[b]{2}{*}{ Characteristics } & \multicolumn{2}{|c|}{ Serum ex-miRNA-21 expression level } & \multirow[b]{2}{*}{ P-value } & \multicolumn{2}{|c|}{ Serum ex-miRNA-210 expression level } & \multirow[b]{2}{*}{ P-value } \\
\hline & $\begin{array}{l}\text { Low expression } \\
\text { group, } \mathrm{n}=12\end{array}$ & $\begin{array}{l}\text { High expression } \\
\text { group, } \mathrm{n}=18\end{array}$ & & $\begin{array}{l}\text { Low expression } \\
\text { group, } n=16\end{array}$ & $\begin{array}{l}\text { High expression } \\
\text { group, }(\mathrm{n}=14)\end{array}$ & \\
\hline Age, $<60 / \geq 60$ years, $\mathrm{n}$ & $5 / 7$ & $7 / 11$ & 0.060 & $5 / 11$ & $6 / 8$ & $0.007^{\mathrm{a}}$ \\
\hline Male/female, $n$ & $8 / 4$ & $12 / 6$ & $>0.999$ & $12 / 4$ & $11 / 3$ & 0.311 \\
\hline Treatment history, No/Yes, n & $10 / 2$ & $15 / 3$ & $0.046^{\mathrm{a}}$ & 9/7 & $8 / 6$ & $0.026^{\mathrm{a}}$ \\
\hline Location, head/body/tail, n & $5 / 3 / 4$ & $5 / 7 / 6$ & $0.010^{\mathrm{a}}$ & $4 / 6 / 6$ & $3 / 5 / 6$ & $0.004^{\mathrm{a}}$ \\
\hline Size of tumor, $<5 / \geq 5 \mathrm{~cm}, \mathrm{n}$ & $7 / 5$ & $10 / 8$ & $0.038^{\mathrm{a}}$ & $10 / 6$ & $8 / 6$ & $0.016^{\mathrm{a}}$ \\
\hline${ }^{\mathrm{b}} \mathrm{T}$ stage, $1-2 / 3-4, \mathrm{n}$ & $4 / 8$ & $7 / 11$ & $0.011^{\mathrm{a}}$ & $16 / 0$ & $12 / 2$ & $0.006^{\mathrm{a}}$ \\
\hline${ }^{\mathrm{b}} \mathrm{N}$ stage, $0 / 1, \mathrm{n}$ & $9 / 3$ & $17 / 1$ & $0.030^{\mathrm{a}}$ & $8 / 8$ & $7 / 7$ & $0.020^{\mathrm{a}}$ \\
\hline${ }^{\mathrm{b}} \mathrm{M}$ stage, $0 / 1, \mathrm{n}$ & $10 / 2$ & $18 / 0$ & 0.250 & $14 / 2$ & $11 / 3$ & 0.201 \\
\hline CA19-9, $<37 / \geq 37, \mathrm{U} / \mathrm{ml}$ & $7 / 5$ & $10 / 8$ & $0.016^{\mathrm{a}}$ & $7 / 9$ & $10 / 4$ & $0.018^{\mathrm{a}}$ \\
\hline $\mathrm{CRP},<10 / \geq 10, \mathrm{mg} / \mathrm{l}$ & $4 / 8$ & $10 / 8$ & $0.020^{\mathrm{a}}$ & $9 / 5$ & $10 / 4$ & $0.013^{\mathrm{a}}$ \\
\hline
\end{tabular}

${ }^{\mathrm{a}} \mathrm{P}<0.05$. ${ }^{\mathrm{b}} \mathrm{TNM}$ stage was determined according to the European Neuroendocrine Tumor Society TNM staging system (33). Fisher's exact test and $\chi^{2}$ tests were used for statistical analysis. CA19-9, carbohydrate antigen 19-9; CRP, C-reactive protein; T, tumor; N, node; M, metastasis.

was diagnostic of pancreatic ductal adenocarcinoma (51). In contrast, miRNA-210 acts as an oncogene, and has been demonstrated to be upregulated in various types of tumors compared with adjacent normal tissues contributing to the progression and development of various cancers, including lung cancer and $\mathrm{PC}$ via different signaling pathways, including the $\mathrm{NF}-\varkappa \mathrm{B}$, hypoxia and Akt- and p53-dependent signaling pathways $(52,53)$. The possible clinical application of miRNA-210 in diagnosing and detecting different types of cancer, such as PC, breast, lung and colorectal cancer, was previously investigated (54). In addition, the serum levels of miRNA-210 were previously found to be induced under hypoxic conditions and linked to adverse prognosis in some types of cancer, including colon and breast carcinoma, esophageal adenocarcinoma and liver cancer (55), as a hypoxic environment is common in PC $(56,57)$, it may be worth investigating the potential diagnostic value of serum ex-miRNAs for patients with PC. The analysis of ex-miRNA-21 and miRNA-210 levels among circulating serum miRNAs may represent a feasible strategy for PC diagnosis. It may be inferred that ex-miRNAs in the serum directly reflect the properties of tumor cells, as well as various diseases as the quantity and content of exosomes may be reflective of the pathophysiological state of the cells (58).

In the present study, the potential diagnostic value of serum ex-miRNAs was compared with the serum levels of CA19-9. Ex-miRNA-21 and miRNA-210 levels may be a new diagnostic or therapeutic target for early PC, while CA19-9 levels remained within the normal range. The sensitivity of the serum test was increased to $90 \%$ when combining positive results for ex-miRNA-21 and miRNA-210 levels with serum CA19-9 levels, while the specificity remained $90 \%$. The findings of the present study revealed the favorable sensitivity and specificity of circulating fr-miRNA-21 and miRNA-210 for the diagnosis of $\mathrm{PC}$, which was consistent with other findings that the high levels of serum/plasma miR-21 and miR-210 could be used as biomarkers for PC $(59,60)$. However, in the present 
study PC-associated secreted ex-miR-21 and miR-210 may have clinical relevance in early detection; higher sensitivity, specificity and accuracy compared with serum fr-miRNA. Exosomes are highly heterogeneous and likely reflect the phenotype of the cell that generate them (61). Similar to cells, exosomes are composed of a lipid bilayer, this structure offers high stability in body fluids, making exosomes highly attractive targets for diagnostic markers (62). Serum exosomes are a valuable tool and different methods may be used to investigate their components, which may lead to more accurate, sensitive, cost-effective, and high-throughput diagnoses (63). The present study demonstrated that the serum ex-miRNA-21 and miRNA-210 levels were closely associated with disease state and other prognostic factors, and that they may be used for early diagnosis and prediction of prognosis non-invasively. These data collectively revealed that these serum exosomal miRNAs are potential valuable biomarkers for the diagnosis, screening and prognosis of PC.

The limitations of the present study included the small sample size, which may affect the diagnostic value of exosomal miRNAs. In addition, the expression level of serum ex-miRNAs was not compared between normal controls and PC tissues i.e., the lack of healthy controls to compare with patients with $\mathrm{PC}$ and $\mathrm{CP}$, and the western blotting data was only qualitative rather than quantitative. The obvious advantage of ex-miRNAs compared with circulating fr-miRNA for the diagnosis of PC will be investigated in depth in future studies. The mechanisms regulating transfer of ex-miRNAs from tumor cells into the blood is rarely reported, while the association between serum and tissue levels of ex-miRNAs should be a focus of future research. In addition, exosomal miRNAs in the plasma, pancreatic juice, bile, urine, saliva, breast milk and other bodily fluids have not yet been investigated as diagnostic biomarkers and will be examined in future studies. Another limitation of the present study was that the level of change of circulating ex-miRNAs was not examined before and after treatment in patients with PC. Finally, the effect of the degree of circulating ex-miRNA expression on disease progression, tumor cell metastasis and overall survival cannot be concluded from the results of the present study due to the small number of patients with PC and the short follow-up period; this limitation must be addressed by future studies.

The present study demonstrated that serum ex-miRNA-21 and miRNA-210 levels distinguished effectively between patients with PC and those with CP. Furthermore, the combination of ex-miRNAs with CA19-9 was more specific and sensitive compared with serum tests alone for the diagnosis of PC. These findings indicate that circulating ex-miRNAs, miRNA-21 and miRNA-210, may be potential novel biomarkers and therapeutic targets for PC. In addition, the quantitation of ex-miRNAs may be used as a clinical examination for further confirmation of diagnosis. Further investigation of a large number of cases is needed to determine the potential diagnostic, prognostic and clinical value of miRNA-21 and miRNA-210 in patients with PC.

\section{Acknowledgements}

Not applicable.

\section{Funding}

The present study was funded by the National Natural Scientific Foundation of China (grant no. 81872509), the Free Exploration Project of Hubei University of Medicine (grant no. FDFR201804), the Hubei Province Health and Family Planning Scientific Research Project (grant no. WJ2019M054), the Natural Science Foundation of the Bureau of Science and Technology of Shiyan City (grant no. 18Y76, 17Y47 and 18Y77) and the Natural Science Foundation of Hubei Provincial Department of Education (grant nos. 2019CFB429 and Q20162113).

\section{Availability of data and materials}

The datasets used and/or analyzed during the current study are available from the corresponding author on reasonable request.

\section{Authors' contributions}

LW, WBZ, QHC and ZGT contributed to experimental design, procedure and data acquisition. LW, JZ, WBZ, ZGT, WW and YW contributed to data analysis, as well as drafting or revising the manuscript. HMW, XDL, XCC, LY and LCY analyzed the data and assisted in the experiments. All authors read and approved the final version of the manuscript.

\section{Ethics approval and consent to participate}

The study protocol was approved by the Ethics Committee of Renmin Hospital of Wuhan University (approval no. 20180212; Wuhan, China). Written informed consent was provided by all patients.

\section{Patient consent for publication}

Not applicable.

\section{Competing interests}

The authors declare that they have no competing interests.

\section{References}

1. Siegel RL, Miller KD and Jemal A: Cancer statistics, 2019. CA Cancer J Clin 69: 7-34, 2019.

2. Qian L, Li Q, Kwaku B, Qiu W, Li K, Zhang J, Yu Q, Xu D, Liu W, Brand RE, et al: Biosensors for early diagnosis of pancreatic cancer: A review. Transl Res 213: 68-89, 2019.

3. Siegel RL, Miller KD and Jemal A: Cancer statistics, 2016. CA Cancer J Clin 66: 7-30, 2016.

4. Park J, Choi Y, Namkung J, Yi SG, Kim H, Yu J, Kim Y, Kwon MS, Kwon W, Oh DY, et al: Diagnostic performance enhancement of pancreatic cancer using proteomic multimarker panel. Oncotarget 8: 93117-93130, 2017.

5. Akamatsu M, Makino N, Ikeda Y, Matsuda A, Ito M, Kakizaki Y, Saito Y, Ishizawa T, Kobayashi T, Furukawa T and Ueno Y: Specific MAPK-Associated MicroRNAs in serum differentiate pancreatic cancer from autoimmune pancreatitis. PLoS One 11: e0158669, 2016.

6. Yu H, Li B, Li T, Zhang S and Lin X: Combination of noninvasive methods in diagnosis of infertile women with minimal or mild endometriosis, a retrospective study in China. Medicine (Baltimore) 98: e16695, 2019. 
7. Zheng $\mathrm{H}$ and Bai L: Hypoxia induced microRNA-301b-3p overexpression promotes proliferation, migration and invasion of prostate cancer cells by targeting LRP1B. Exp Mol Pathol 20: 104301, 2019.

8. Fadaka AO, Pretorius A and Klein A: Biomarkers for Stratification in Colorectal Cancer: MicroRNAs. Cancer Control 26: 1073274819862784, 2019.

9. Takagawa Y, Gen Y, Muramatsu T, Tanimoto K, Inoue J, Harada H and Inazawa J: miR-1293, a Candidate for miRNA-based cancer therapeutics, simultaneously targets BRD4 and the DNA repair pathway. Mol Ther 11. pii: S1525-0016: 30182-30189, 2020.

10. Örs Kumoğlu G, Döşkaya M and Gulce Iz S: The biomarker features of miR-145-3p determined via meta-analysis validated by qRT-PCR in metastatic cancer cell lines. Gene 710: 341-353, 2019.

11. Li Z, Meng Q, Pan A, Wu X, Cui J, Wang Y and Li L: MicroRNA-455-3p promotes invasion and migration in triple negative breast cancer by targeting tumor suppressor EI24. Oncotarget 8: 19455-19466, 2017.

12. Bai X, Lu D, Lin Y, Lv Y and He L: A seven-miRNA expression-based prognostic signature and its corresponding potential competing endogenous RNA network in early pancreatic cancer. Exp Ther Med 18: 1601-1608, 2019

13. Kooshkaki O, Rezaei Z, Rahmati M, Vahedi P, Derakhshani A, Brunetti O, Baghbanzadeh A, Mansoori B, Silvestris N and Baradaran B: MiR-144: A new possible therapeutic target and diagnostic/prognostic tool in cancers. Int J Mol Sci 21: pii: E2578, 2020.

14. Aggarwal V, Priyanka K and Tuli HS: Emergence of circulating MicroRNAs in breast cancer as diagnostic and therapeutic efficacy biomarkers. Mol Diagn Ther 24: 153-173, 2020.

15. Valadi H, Ekström K, Bossios A, Sjöstrand M, Lee JJ and Lötvall JO: Exosome-mediated transfer of mRNAs and microRNAs is a novel mechanism of genetic exchange between cells. Nat Cell Biol 9: 654-659, 2007.

16. Tang Y,Zhao Y, Song X, Song X, Niu L and Xie L: Tumor-derived exosomal miRNA-320d as a biomarker for metastatic colorectal cancer. J Clin Lab Anal 16: e23004, 2019.

17. Munson PB, Hall EM, Farina NH, Pass HI and Shukla A: Exosomal miR-16-5p as a target for malignant mesothelioma. Sci Rep 9: 11688, 2019.

18. Sun Z, Shi K, Yang S, Liu J, Zhou Q, Wang G, Song J, Li Z, Zhang Z and Yuan W: Effect of exosomal miRNA on cancer biology and clinical applications. Mol Cancer 17: 147, 2018

19. Wu H, Chen X, Ji J, Zhou R, Liu J, Ni W, Qu L, Ni H, Ni R, Bao B and Xiao M: Progress of exosomes in the diagnosis and treatment of pancreatic cancer. Genet Test Mol Biomarkers 23: 215-222, 2019.

20. Bortoluzzi S, Lovisa F, Gaffo E and Mussolin L: Small RNAs in circulating exosomes of cancer patients: A minireview. High Throughput 6: pii: E13, 2017.

21. Fanale D, Taverna S, Russo A and Bazan V: Circular RNA in exosomes. Adv Exp Med Biol 1087: 109-117, 2018.

22. Jayaseelan VP: Emerging role of exosomes as promising diagnostic tool for cancer. Cancer Gene Ther: Sep 3, 2019 (Epub ahead of print).

23. Wang W, Yin Y, Shan X, Zhou X, Liu P, Cao Q, Zhu D, Zhang J and Zhu W: The value of plasma-based MicroRNAs as diagnostic biomarkers for ovarian cancer. Am J Med Sci 358: 256-267, 2019

24. He D, Wang H, Ho SL, Chan HN, Hai L, He X, Wang K and Li HW: Total internal reflection-based single-vesicle in situ quantitative and stoichiometric analysis of tumor-derived exosomal microRNAs for diagnosis and treatment monitoring. Theranostics 9: 4494-4507, 2019.

25. Bai H, Lei K, Huang F, Jiang Z and Zhou X: Exo-circRNAs: A new paradigm for anticancer therapy. Mol Cancer 18: 56, 2019.

26. Yi M, Xu L, Jiao Y, Luo S, Li A and Wu K: The role of cancer-derived microRNAs in cancer immune escape. J Hemato Oncol 13: 25, 2020

27. Nguyen HV, Gore J, Zhong X, Savant SS, Deitz-McElyea S, Schmidt CM, House MG and Korc M: MicroRNA expression in a readily accessible common hepatic artery lymph node predicts time to pancreatic cancer recurrence postresection. J Gastrointest Surg 20: 1699-706, 2016.

28. Xie Y, Hang Y, Wang Y, Sleightholm R, Prajapati DR, Bader J, Yu A, Tang W, Jaramillo L, Li J, et al: Stromal modulation and treatment of metastatic pancreatic cancer with local intraperitoneal triple miRNA/siRNA nanotherapy. ACS Nano 14: 255-271, 2020
29. Yang Z, LaRiviere MJ, Ko J, Till JE, Christensen T, Yee SS, Black TA, Tien K, Lin A, Shen H, et al: A multi-analyte panel consisting of extracellular vesicle miRNAs and mRNAs, cfDNA, and CA19-9 shows utility for diagnosis and staging of pancreatic adenocarcinoma. Clin Cancer Res 16: pii: clincanres 3313: 2019, 2020.

30. Yoshizawa N, Sugimoto K, Tameda M, Inagaki Y, Ikejiri M, Inoue $\mathrm{H}$, Usui $\mathrm{M}$, Ito $\mathrm{M}$ and Takei $\mathrm{Y}$ : $\mathrm{miR}-3940-5 \mathrm{p} / \mathrm{miR}-8069$ ratio in urine exosomes is a novel diagnostic biomarker for pancreatic ductal adenocarcinoma. Oncol Lett 19: 2677-2684, 2020.

31. Ko J, Bhagwat N, Black T, Yee SS, Na YJ, Fisher S, Kim J, Carpenter EL, Stanger BZ and Issadore D: miRNA profiling of magnetic nanopore-isolated extracellular vesicles for the diagnosis of pancreatic cancer. Cancer Res 78: 3688-3697, 2018.

32. Matsuda Y, Fujii Y, Matsukawa M, Ishiwata T, Nishimura M and Arai T: Overexpression of carbohydrate sulfotransferase 15 in pancreatic cancer stroma is associated with worse prognosis. Oncol Lett 18: 4100-4105, 2019.

33. Luo G, Javed A, Strosberg JR, Jin K, Zhang Y, Liu C, Xu J, Soares K, Weiss MJ, Zheng L, et al: Modified staging classification for pancreatic neuroendocrine tumors on the basis of the American joint committee on cancer and european neuroendocrine tumor society systems. J Clin Oncol 35: 274-280, 2017.

34. Lee YR, Kim G, Tak WY, Jang SY, Kweon YO, Park JG, Lee HW, Han YS, Chun JM, Park SY and Hur K: Circulating exosomal noncoding RNAs as prognostic biomarkers in humanhepatocellular carcinoma. Int J Cancer 144: 1444-1452, 2019.

35. Shi M, Jiang Y, Yang L, Yan S, Wang YG and Lu XJ: Decreased levels of serum exosomal miR-638 predict poor prognosis in hepatocellular carcinoma. J Cell Biochem 119: 4711-4716, 2018.

36. Suehiro T, Miyaaki H, Kanda Y, Shibata H, Honda T, Ozawa E, Miuma S, Taura N and Nakao K: Serum exosomal microRNA-122 and microRNA-21 as predictive biomarkers in transarterial chemoembolization-treated hepatocellular carcinoma patients. Oncol Lett 16: 3267-3273, 2018.

37. Shiotsu H, Okada K, Shibuta T, Kobayashi Y, Shirahama S, Kuroki C, Ueda S, Ohkuma M, Ikeda K, Ando Y, et al: The influence of pre-analytical factors on the analysis of circulating MicroRNA. Microrna 7: 195-203, 2018

38. Livak KJ and Schmittgen TD: Analysis of relative gene expression data using real-time quantitative PCR and the 2(-Delta Delta C(T)) method. Methods 25: 402-408, 2001.

39. Silverman JM and Reiner NE: Exosomes and other microvesicles in infection biology: Organelles with unanticipated phenotypes. Cell Microbiol 13: 1-9, 2011.

40. Rahbarghazi R, Jabbari N, Sani NA, Asghari R, Salimi L, Kalashani SA, Feghhi M,Etemadi T, Akbariazar E, Mahmoudi M and Rezaie J: Tumor-derived extracellular vesicles: Reliable tools for Cancer diagnosis and clinical applications. Cell Commun Signal 17: 73, 2019.

41. Salehi M and Sharifi M: Exosomal miRNAs as novel cancer biomarkers: Challenges and opportunities. J Cell Physiol 233: 6370-6380, 2018

42. Wang M, Yu F, Ding H, Wang Y, Li P and Wang K: Emerging function and clinical values of exosomal MicroRNAs in cancer. Mol Ther Nucleic Acids 16: 791-804, 2019.

43. Capula M, Mantini G, Funel N and Giovannetti E: New avenues in pancreatic cancer: Exploiting microRNAs as predictive biomarkers and new approaches to target aberrant metabolism. Expert Rev Clin Pharmacol 12: 1081-1090, 2019.

44. Taylor DD and Gercel-Taylor C: MicroRNA signatures of tumor-derived exosomes as diagnostic biomarkers of ovarian cancer. Gynecol Oncol 110: 13-21, 2008.

45. Rabinowits G, Gerçel-Taylor C, Day JM, Taylor DD and Kloecker GH: Exosomal microRNA: A diagnostic marker for lung cancer. Clin Lung Cancer 10: 42-46, 2009.

46. Sohn W, Kim J, Kang SH, Yang SR, Cho JY, Cho HC, Shim SG and Paik YH: Serum exosomal microRNAs as novel biomarkers for hepatocellular carcinoma. Exp Mol Med 47: e184, 2015.

47. Valadi H, Ekström K, Bossios A, Sjöstrand M, Lee JJ and Lötvall JO: Exosome mediated transfer of mRNAs and microRNAs is a novel mechanism of genetic exchange between cells. Nat Cell Biol 9: 654-659, 2007.

48. de Carvalho IN, de Freitas RM and Vargas FR: Translating microRNAs into biomarkers: What is new for pediatric cancer? Med Oncol 33: 49, 2016.

49. Su J, Wu F, Xia H, Wu Y and Liu S: Accurate cancer cell identification and microRNA silencing induced therapy using tailored DNA tetrahedron nanostructures. Chem Sci 11: 80-86, 2019. 
50. Luo D, Huang Z, Lv H, Wang Y, Sun W and Sun X: Up-regulation of MicroRNA-21 indicates poor prognosis and promotes cell proliferation in esophageal squamous cell carcinoma via upregulation of lncRNA SNHG1. Cancer Manag Res 12: 1-14, 2020.

51. Goto T, Fujiya M, Konishi H, Sasajima J, Fujibayashi S, Hayashi A, Utsumi T, Sato H, Iwama T, Ijiri M, et al: An elevated expression of serum exosomal microRNA-191, - 21, -451a of pancreatic neoplasm is considered to be efficient diagnostic marker. BMC Cancer 18: 116, 2018

52. Feng S, He A, Wang D and Kang B: Diagnostic significance of miR-210 as a potential tumor biomarker of human cancer detection: An updated pooled analysis of 30 articles. Onco Targets Ther 12: 479-493, 2019.

53. Hong L, Han Y, Zhang H, Zhao Q and Qiao Y: miR-210: A therapeutic target in cancer. Expert Opin Ther Targets 17: 21-28, 2013

54. Ren CX, Leng RX, Fan YG, Pan HF, Wu CH and Ye DQ: MicroRNA-210 and its theranostic potential. Expert Opin Ther Targets 20: 1325-1338, 2016

55. Bavelloni A, Ramazzotti G, Poli A, Piazzi M, Focaccia E, Blalock W and Faenza I: MiRNA-210: A current overview. Anticancer Res 37: 6511-6521, 2017.

56. Li W, Liu H, Qian W, Cheng L, Yan B, Han L, Xu Q, Ma Q and Ma J: Hyperglycemia aggravates microenvironment hypoxia and promotes the metastatic ability of pancreatic cancer. Comput Struct Biotechnol J 16: 479-487, 2018.

57. Chen S, Zhang J, Chen J, Wang Y, Zhou S, Huang L, Bai Y, Peng C, Shen B, Chen $\mathrm{H}$ and Tian Y: RER1 enhances carcinogenesis and stemness of pancreatic cancer under hypoxic environment. J Exp Clin Cancer Res 38: 15, 2019.
58. Severino V, Dumonceau JM, Delhaye M, Moll S, Annessi-Ramseyer I, Robin X, Frossard JL and Farina A: Extracellular vesicles in bile as markers of malignant biliary stenoses. Gastroenterology 153: 495-504.e8, 2017.

59. Wang J, Chen J, Chang P, LeBlanc A, Li D, Abbruzzesse JL, Frazier ML, Killary AM and Sen S: MicroRNAs in plasma of pancreatic ductal adenocarcinoma patients as novel blood-based biomarkers of disease. Cancer Prev Res 2: 807-813, 2009

60. Qu K, Zhang X, Lin T, Liu T, Wang Z, Liu S, Zhou L, Wei J, Chang H, Li K, et al: Circulating miRNA-21-5p as a diagnostic biomarker for pancreatic cancer: Evidence from comprehensive miRNA expression profiling analysis and clinical validation. Sci Rep 7: 1692, 2017.

61. Kowal J, Arras G, Colombo M, Jouve M, Morath JP, Primdal-Bengtson B, Dingli F, Loew D, Tkach M and Thery C: Proteomic comparison defines novel markers to characterize heterogeneous populations of extracellular vesicle subtypes. Proc Natl Acad Sci USA 113: E968-E977, 2016.

62. Hu Q, Su H, Li J, Lyon C, Tang W, Wan M and Hu TY: Clinical applications of exosome membrane proteins. Precis Clin Med 3: 54-66, 2020.

63. Makler A and Asghar W: Exosomal biomarkers for cancer diagnosis and patient monitoring. Expert Rev Mol Diagn 20: $387-400,2020$.

(i) $($ This work is licensed under a Creative Commons EY NO ND Attribution-NonCommercial-NoDerivatives 4.0 International (CC BY-NC-ND 4.0) License. 